\title{
Hyperinsulinemic hypoglycemia associated with insulin antibodies caused by exogenous insulin analog
}

\author{
Chih-Ting Su ${ }^{1}$ and Yi-Chun Lin ${ }^{1,2}$ \\ ${ }^{1}$ Faculty of Medicine, National Yang-Ming University, Taipei, Taiwan and 2Department of Medicine, \\ Division of Endocrinology and Metabolism, Taipei Veterans General Hospital, Taipei, Taiwan
}

Correspondence should be addressed to Y-C Lin

Email

yclin15@vghtpe.gov.tw

\section{Summary}

Insulin antibodies (IA) associated with exogenous insulin administration seldom caused hypoglycemia and had different characteristics from insulin autoantibodies (IAA) found in insulin autoimmune syndrome (IAS), which was first described by Dr Hirata in 1970. The characteristic of IAS is the presence of insulin-binding autoantibodies and related fasting or late postprandial hypoglycemia. Here, we report a patient with type 1 diabetes mellitus under insulin glargine and insulin aspart treatment who developed recurrent spontaneous post-absorptive hyperinsulinemic hypoglycemia with the cause probably being insulin antibodies induced by exogenous injected insulin. Examinations of serial sera disclosed a high titre of insulin antibodies ( $33 \%$, normal $<5 \%)$, high insulin concentration $(111.9 \mathrm{IU} / \mathrm{mL})$ and undetectable C-peptide when hypoglycemia occurred. An oral glucose tolerance test revealed persistent high serum levels of total insulin and undetectable C-peptide. Image studies of the pancreas were unremarkable, which excluded the diagnosis of insulinoma. The patient does not take any of the medications containing sulfhydryl compounds, which had been reported to cause IAS. After administering oral prednisolone for 3 weeks, hypoglycemic episodes markedly improved, and he was discharged smoothly.

\section{Learning points:}

- Insulin autoimmune syndrome (IAS) or IAS-like situation should be one of the differential diagnosis in patients with hyperinsulinemic hypoglycemia.

- Although less reported, insulin antibodies (IA) caused by exogenous insulin analog should be considered as the cause of hypoglycemia.

- Patients with suspected insulin autoimmune syndrome (IAS) should be screened for drugs related to autoimmunity to endogenous insulin.

\section{Background}

Hyperinsulinemic hypoglycemia can be caused by exogenous or endogenous insulin action. As a gold standard test for hypoglycemia, prolonged fast test helps to confirm the presence of inappropriate insulin secretion when hypoglycemia developed. The differential diagnosis of hyperinsulinemic hypoglycemia included insulin injection, sulfonylurea drug, insulinoma, nesidioblastosis and insulin autoantibodies (IAA)-related insulin autoimmune syndrome (IAS). Insulin autoimmune syndrome (IAS), which is characterized by a high concentration of total serum immune-reactive insulin (IRI) and circulating insulin autoantibodies (IAA) during 
hypoglycemia, should be considered after excluding insulinoma and nesidioblastosis $(1,2,3)$. Patients with IAS often have underlying autoimmune disorders or have been exposed to certain drugs containing a sulfhydryl group, such as methimazole. In rare cases, insulin antibodies (IA) associated with exogenous insulin administration caused hypoglycemia. Insulin antibodies (IA) are often seen in patients receiving insulin treatment. These insulin antibodies (IA), which typically had low capacity and high affinity rarely cause hypoglycemia, which are unlike the high-capacity and low-affinity characteristics of IAA presented in IAS $(3,4)$. Herein is a rare case of IA-related recurrent severe hypoglycemic coma probably associated with exogenous insulin analogs, which had similar clinical presentation as IAS.

\section{Case presentation}

A 48-year-old man was admitted to our hospital with recurrent severe hypoglycemic coma. His history included type 1 diabetes mellitus for 20 years, receiving multipledose insulin injection. Initially, he received Humulin $\mathrm{R}$ and Humulin $\mathrm{N}$ injection with total daily dose about 40 units. He started to use insulin aspart plus insulin glargine since 4 years ago and latest hemoglobin A1c was $7.6 \%$. Frequent hypoglycemia and syncope events were noted for 1 year. At first, hypoglycemic coma happened about twice annually, but the frequency increased to 3 times a week in recent months. In the past three months, the patient repetitively suffered from unconsciousness and was sent to the emergency department of hospitals nearby where severe hypoglycemia below $30 \mathrm{mg} / \mathrm{dL}$ was noted. He was referred to our hospital for further survey of etiology of hypoglycemia. During hospitalization, several episodes of disturbed consciousness due to hypoglycemia recurred. Clinically, the patient had clear consciousness most of the time but looked chronically ill, with a body weight of $54 \mathrm{~kg}$. He denied an exposure history to a sulfhydryl drugs, such as methimazole.

\section{Investigation}

On physical examination, his body height was $163 \mathrm{~cm}$, weighed $54 \mathrm{~kg}$ and body mass index was 20 , others disclosed no significant finding. Blood cell counts and biochemical data were unremarkable.

During one episode of disturbed consciousness, the following values were recorded: serum glucose concentration: $34 \mathrm{mg} / \mathrm{dL}$; insulin (IRI): $61.1 \mathrm{IU} / \mathrm{mL}$; C-peptide $<0.01 \mathrm{ng} / \mathrm{mL}$. The patient was euthyroid with thyroid-stimulating hormone (TSH) level of $0.32 \mathrm{IU} / \mathrm{mL}$ and free T4 level of $0.92 \mathrm{IU} / \mathrm{mL}$. For survey of adrenal insufficiency, the $08: 00 \mathrm{~h}$ serum cortisol level was $11.1 \mu \mathrm{g} / \mathrm{dL}$. Due to high insulin but low C-peptide level, factitious disorder was first suspected. We had ensured that he did not self-administrate insulin during hospitalization by frequent visits by nurses especially during sleep time.

Physician-attended prolonged fast test was performed, and hypoglycemia episode developed soon after $4 \mathrm{~h}$ with hyperinsulinemia with the following values: serum glucose concentration: $38 \mathrm{mg} / \mathrm{dL}$; insulin (IRI): $111.9 \mathrm{IU} / \mathrm{mL}$ and C-peptide: $<0.01 \mathrm{ng} / \mathrm{mL}$. These findings were consistent with hyperinsulinemic hypoglycemia. In addition, an unusually high level of insulin antibodies (33\%; normal, <5\%) was identified in the patient's serum. Computed tomography of the abdomen was negative.

Capillary blood glucose concentrations were monitored before meals, before bed time and at 03:00 $\mathrm{h}$ to study the pattern of hypoglycemia in the patient. A 24-h blood glucose profile revealed a fasting hypoglycemia, with a nadir frequently occurring at around 05:00-06:00 h of a day (Fig. 1). To further define the relationship between the serum concentrations of glucose and total insulin, an oral glucose tolerance test was performed. After a $75 \mathrm{~g}$ glucose load, total insulin level increased markedly at $30 \mathrm{~min}$, but it was kept in high level greater than $80 \mathrm{IU} / \mathrm{mL}$. At the time point of $5 \mathrm{~h}$, hypoglycemia with blood glucose of $45 \mathrm{mg} / \mathrm{dL}$ and a simultaneous insulin level of 97.9 IU/mL were recorded, which indicated postabsorptive hyperinsulinemic hypoglycemia (Fig. 2).

\section{Treatment}

Insulin regimens were shifted from insulin aspart plus insulin glargine to Humulin $\mathrm{N}$ and Humulin R 2 days after admission. However, the severity and frequency of

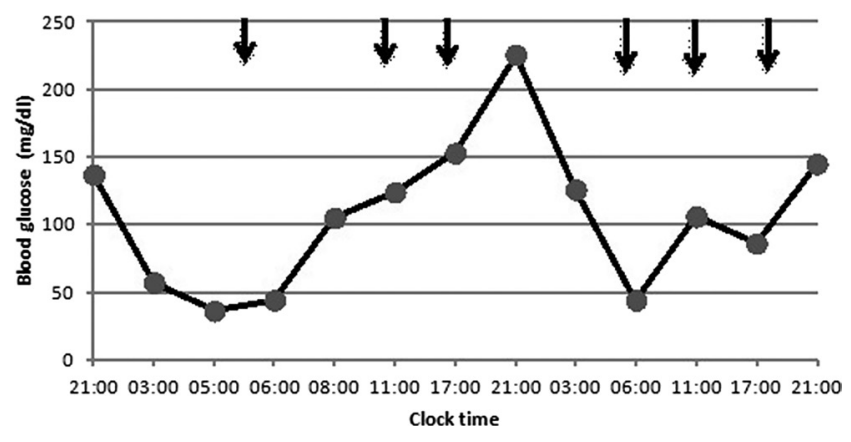

Figure 1

A 48-h blood glucose profile revealed early morning hypoglycemia. Black arrows indicate meal ingestion. 


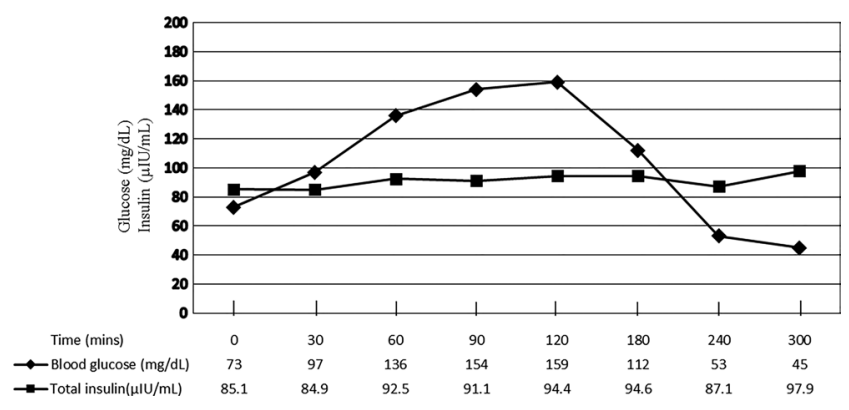

Figure 2

After a $75 \mathrm{~g}$ glucose load, total insulin remained in high level until $5 \mathrm{~h}$, indicating a relatively delayed clearance. At $5 \mathrm{~h}$ after load, blood glucose $45 \mathrm{mg} / \mathrm{dL}$ with insulin $97.9 \mathrm{IU} / \mathrm{mL}$. Glucose (solid diamonds) and total insulin (solid squares).

hypoglycemia events did not improve. Prednisolone was then administered one week later with the dosage of $30 \mathrm{mg}$ per day for the following 3 weeks during hospitalization.

\section{Outcome and follow-up}

After administering oral prednisolone $30 \mathrm{mg}$ per day, the severity and frequency of hypoglycemia events improved in one week. The time of nadir blood glucose level during a day is more predictably with hypoglycemia frequency decreased from once or twice a day to twice a week during the following weeks. It usually happened around 3 o'clock to 6 o'clock in early morning with a glucose level greater than $60 \mathrm{mg} / \mathrm{dL}$. No liable glucose control was noted after prednisolone treatment by blood glucose monitoring 4 times a day. We kept his insulin regimen with multiple dose injection of Humulin R and Humulin $\mathrm{N}$ during this period. He was then discharged smoothly with $30 \mathrm{mg}$ oral prednisolone per day.

\section{Discussion}

Insulin antibodies (IA) are not uncommon in patients receiving insulin injection. These antibodies had the characteristics of low capacity and high affinity, which seldom cause hypoglycemia or hyperglycemia (5). From the literature, incidence of $0.16 \%$ of exogenous insulininduced insulin antibodies in patients with diabetes had been reported (6). In a study surveying 118 Japanese patients who used insulin, insulin antibodies were detected in $40.7 \%$ of them, and insulin glargine was the most antigenic (7). Insulin analog has an amino acid sequence different from human insulin, which could possibly be recognized as an antigen. On the other hand, insulin autoimmune syndrome (IAS), or Hirata disease, is a rare cause of hyperinsulinemic hypoglycemia characterized by autoantibodies to insulin (IAA) caused by medications other than exogenous insulin (8). The antibodies seen in the disease had high capacity and low affinity and lead to hypoglycemia. It is the third leading cause of spontaneous hypoglycemia in Japan after insulinoma and extra-pancreatic neoplasms. About 400 cases have been reported in the medical literature since, with the majority (90\%) depicted in the Japanese population (9). An underlying autoimmune disease or an exposure history to a sulfhydryl drug, such as methimazole, is found associated with IAS. Our patient had not taken sulfhydrylcontaining drugs or other drugs reportedly associated with IAS. Tracing back his treatment of type 1 diabetic mellitus, there were four types of insulin: Humulin N, Humulin R, insulin aspart and insulin glargine. Whether the insulin antibodies are caused by insulin analogs or humulin insulin cannot be documented in these patients for the reason that he did not had the insulin antibodies checked before or during the 20 years of disease. However, according to the temporal association that hypoglycemia events became obvious after shifting to insulin analogs and the higher immunogenic effect of insulin glargine than human insulin in the literature (7), insulin analogs would be the first priority to consider. Therefore, IA induced by exogenous insulin that had the characteristics of high capacity and low affinity was the most likely cause of hypoglycemia in this patient.

In our patient, the markedly elevated levels of total insulin, low C-peptide level, high level of insulin antibodies (33\%) together with the fact that total insulin remained elevated while plasma glucose began to decline during an oral glucose tolerance test, suggest a delayed clearance of insulin, apparently due to binding of released insulin by insulin-binding antibodies. The notion that the insulin-antibody complex is responsible for the development of late postprandial hypoglycemia similar to patients with IAS was introduced by Uchigata et al. (9). There are some case reports about IAS-like situation induced by exogenous insulin. Reported in 2014, a lean 15 -year-old girl was diagnosed with type 1 diabetes based on symptomatic hyperglycemia and positive anti-islet cell antibodies required for treatment of hyperglycemia and ketosis alternating with refractory hypoglycemia after using twice-daily mixed insulin for 11 months (10). A case report of two patients who developed daytime hyperglycemia and early morning hypoglycemia because of insulin antibody (IA) with affinity lower and binding capacity higher than insulin autoantibodies (IAA) in 
the insulin autoimmune syndrome (IAS) after insulin treatment being changed from human insulin to analog insulin was published in 2009 (3).

The largest case series of insulin antibody-associated immunological hypoglycemia was an 11 cases report from China (11). The conclusion is that hypoglycemia associated with insulin antibodies shall not be neglected in insulin-treated patients and if unexpectedly recurrent hypoglycemia occurred, especially after insulin had been discontinued for several days, immunological hypoglycemia associated with IA due to exogenous insulin should be considered (11). The authors concluded that prompt insulin withdrawal was needed; however, this was not possible in our patient who had type 1 diabetes mellitus. Recurrent hyperinsulinemic hypoglycemia would disappear after discontinuing drugs causing IAS (12). In patients with prolonged recurrent hyperinsulinemic hypoglycemia or unknown cause of IAS, immunosuppressive therapy may help to shorten the duration of the disease. Corticosteroid and plasmapheresis are usually the choices of immunosuppressive therapy. Unfortunately, this patient had type 1 diabetes and should be treated with multiple doses of insulin injection without cessation. Therefore, he took oral prednisolone $30 \mathrm{mg}$ per day for his IAS-like situation, and a good response was observed without the deterioration of hyperglycemia by frequent blood sugar monitoring for 2 weeks in the hospital, which is similar to previous case reports $(3,13)$.

Mostly, the hypoglycemic episodes happened during the late postprandial period, and it was assumed that most insulin secreted from endogenous origin or administered from exogenous method was bound to antibodies and then dissolved from the complex to exert its effect, thus producing postprandial hypoglycemia $(14,15)$. IAS can present with fasting or post-absorptive hypoglycemia. In our patient, the hypoglycemia usually developed at early morning or post-absorptive status (Figs 1 and 2), which is consistent with typical IAS. The triggering factors that cause the dissociation of insulin and insulin antibodies are not clear.

The cause of inducing insulin antibodies (IA) is heterogeneous and remains incompletely understood. From the study of IAS, underlying autoimmune disorders, specific human leukocyte antigen (HLA) typing, sulfhydryl-containing drugs such as methimazole, thiopronin, penicillamine, glutathione and other drugs, including hydralazine, procainamide and isoniazid, have been associated with this uncommon syndrome
$(8,16,17,18,19)$. Immunogenetic evidence suggests a striking association between polyclonal IAS and specific HLA class II antigens such as DRB1*0406, DQA1*0301 and DQB1*0302 $(1,3,17)$. Further studies indicate that certain amino acids at position 74 in the HLA-DR4 $\beta 1$ chain might be involved in the pathogenesis of insulin autoantibody production (17). Other factors attributed to IA production included purity and species of insulin administrated (20).

The major limitation of current case report is the lack of Scatchard plot analysis. The IA emerged from insulin analogs with high capacity and low affinity should be proven by characterizing these different clones of antibodies. The case presented here wish to raise the attention to the possibility that IA induced by exogenous insulin analogs may have the similar clinical characteristics as insulin autoantibodies (IAA) seen in IAS and should be considered in diabetes patients treated with insulin. Further, Scatchard analysis of the IA is warranted to establish the exact clones and prevalence of IA that will affect pharmacokinetics of insulin therapy.

In conclusion, IA, although uncommon clinically, should be one of the differential diagnosis in patients with hyperinsulinemic hypoglycemia. IA induced by exogenous insulin analog seldom had clinical significance but still causes hypoglycemia in our case and in few case reports which had the similar clinical effects as IAS. This should be considered in patients having type 1 diabetes mellitus with frequent hyperinsulinemia hypoglycemia

Declaration of interest

The authors declare that there is no conflict of interest that could be perceived as prejudicing the impartiality of the research reported.

\section{Funding}

This research did not receive any specific grant from any funding agency in the public, commercial or not-for-profit sector.

\section{Patient consent}

Written informed consent has been obtained from the patient.

Author contribution statement

Su C T wrote the article. Lin Y C contributed to the writing and discussion of the article and was the named physician involved in care of the patient. 


\section{References}

1 Cavaco B, Uchigata Y, Porto T, Amparo-Santos M, Sobrinho L \& Leite V 2001 Hypoglycaemia due to insulin autoimmune syndrome: report of two cases with characterisation of HLA alleles and insulin autoantibodies. European Journal of Endocrinology 145 311-316. (doi:10.1530/eje.0.1450311)

2 Uchigata Y, Tokunaga K, Nepom G, Bannai M, Kuwata S, Dozio N, Benson EA, Ronningen KS, Spinas GA, Tadokoro K, et al. 1995 Differential immunogenetic determinants of polyclonal insulin autoimmune syndrome (Hirata's disease) and monoclonal insulin autoimmune syndrome. Diabetes 44 1227-1232. (doi:10.2337/ diab.44.10.1227)

3 Ishizuka T, Ogawa S, Mori T, Nako K, Nakamichi T, Oka Y \& Ito S 2009 Characteristics of the antibodies of two patients who developed daytime hyperglycemia and morning hypoglycemia because of insulin antibodies. Diabetes Research and Clinical Practice 84 e21-e23. (doi:10.1016/j.diabres.2009.02.007)

4 Matsuyoshi A, Shimoda S, Tsuruzoe K, Taketa K, Chirioka T, Sakamoto F, Sakakida M, Miyamura N \& Araki E 2006 A case of slowly progressive type 1 diabetes with unstable glycemic control caused by unusual insulin antibody and successfully treated with steroid therapy. Diabetes Research and Clinical Practice 72 238-243. (doi:10.1016/j.diabres.2005.10.018)

5 Wredling R, Lins PE \& Adamson U 1990 Prevalence of antiinsulin antibodies and its relation to severe hypoglycaemia in insulin-treated diabetic patients. Scandinavian Journal of Clinical and Laboratory Investigation 50 551-557. (doi:10.1080/00365519009089170)

6 Van Haeften TW 1989 Clinical significance of insulin antibodies in insulin-treated diabetic patients. Diabetes Care 12 641-648. (doi:10.2337/diacare.12.9.641)

7 Hattori N, Duhita MR, Mukai A, Matsueda M \& Shimatsu A 2014 Development of insulin antibodies and changes in titers over a longterm period in patients with type 2 diabetes. Clinica Chimica Acta 2014433 135-138. (doi:10.1016/j.cca.2014.03.008)

8 Goldman J, Baldwin D, Pugh W \& Rubenstein AH 1978 Equilibrium binding assay and kinetic characterization of insulin antibodies. Diabetes 27 653-660. (doi:10.2337/diab.27.6.653)

9 Uchigata Y \& Hirata Y 1999 Insulin autoimmune syndrome (IAS, Hirata disease). Annales de Médecine Interne 150 245-253.
10 Jassam N, Amin N, Holland P, Semple RK, Halsall DJ, Wark G \& Barth JH 2014 Analytical and clinical challenges in a patient with concurrent type 1 diabetes, subcutaneous insulin resistance and insulin autoimmune syndrome. Endocrinology, Diabetes and Metabolism Case Reports 2014 130086. (doi:10.1530/EDM-13-0086)

11 Quan H, Tan H, Li Q, Li J \& Li S 2015 Immunological hypoglycemia associated with insulin antibodies induced by exogenous insulin in 11 Chinese patients with diabetes. Journal of Diabetes Research 2015 746271. (doi:10.1155/2015/746271)

12 Roh E, Kim YA, Ku EJ, Bae JH, Kim HM, Cho YM, Park YJ, Park KS, Kim SY \& Kwak SH 2013 Two cases of Methimazole-induced insulin autoimmune syndrome in Graves' disease. Endocrinology and Metabolism 28 55-60. (doi:10.3803/EnM.2013.28.1.55)

13 Koyama R, Nakanishi K, Kato M, Yamashita S, Kuwahara H \& Katori H 2005 Hypoglycemia and hyperglycemia due to insulin antibodies against therapeutic human insulin: treatment with double filtration plasmapheresis and prednisolone. American Journal of the Medical Sciences 329 259-264.14. (doi:10.1097/00000441-200505000-00007)

14 Seino S, Fu ZZ, Marks W, Seino Y, Imura H \& Vinik A 1986 Characterization of circulating insulin in insulin autoimmune syndrome. Journal of Clinical Endocrinology and Metabolism 62 64-69. (doi:10.1210/jcem-62-1-64)

15 Wang YL, Yao PW, Zhang XT, Luo ZZ, Wu PQ \& Xiao F 2015 Insulin autoimmune syndrome: 73 cases of clinical analysis. Chinese Medical Journal 128 2408-2409. (doi:10.4103/0366-6999.163376)

16 Ma WY, Won JG, Tang KT \& Lin HD 2005 Severe hypoglycemic coma due to insulin autoimmune syndrome. Journal of the Chinese Medical Association 68 82-86. (doi:10.1016/S1726-4901(09)70140-6)

17 Benson EA, Healey LA \& Barron EJ 1985 Insulin antibodies in patients receiving penicillamine. American Journal of Medicine 78 857-860. (doi:10.1016/0002-9343(85)90294-3)

18 Burch HB, Clement S, Sokol MS \& Landry F 1992 Reactive hypoglycemic coma due to insulin autoimmune syndrome: case report and literature review. American Journal of Medicine 92 681-685. (doi:10.1016/0002-9343(92)90787-C)

19 Fineberg SE, Kawabata TT, Finco-Kent D, Fountaine RJ, Finch GL \& Krasner AS 2007 Immunological responses to exogenous insulin. Endocrine Reviews 28 625-652. (doi:10.1210/er.2007-0002)

20 Van Haeften TW 1989 Clinical significance of insulin antibodies in insulin-treated diabetic patients. Diabetes Care 12 641-648. (doi:10.2337/diacare.12.9.641)

Received in final form 12 October 2016

Accepted 2 November 2016 